\title{
Correction to: COVID-19 is not "just another flu": a real-life comparison of severe COVID-19 and influenza in hospitalized patients in Vienna, Austria
}

\author{
Erich Pawelka ${ }^{1}$ (1) - Mario Karolyi ${ }^{1}$ - Theresa Mader ${ }^{1} \cdot$ Sara Omid $^{1} \cdot$ Hasan Kelani ${ }^{1} \cdot$ Sebastian Baumgartner ${ }^{1}$. \\ Sarah Ely ${ }^{2}$. Wolfgang Hoepler ${ }^{1} \cdot$ Bernd Jilma $^{2}$. Franz Koenig ${ }^{3} \cdot$ Hermann Laferl $^{1} \cdot$ Marianna Traugott $^{1}$. \\ Michael Turner ${ }^{1} \cdot$ Tamara Seitz $^{1} \cdot$ Christoph Wenisch $^{1} \cdot$ Alexander Zoufaly $^{1}$
}

Published online: 21 July 2021

(c) Springer-Verlag GmbH Germany, part of Springer Nature 2021

\section{Correction to: Infection https://doi.org/10.1007/s15010-021-01610-z}

The original version of this article unfortunately contained a mistake.

In the original version of this article, the given and family names of all author names were incorrectly structured. The corrected author list is given above.

The original article has been corrected.

The original article can be found online at https://doi.org/10.1007/ s15010-021-01610-z.

Erich Pawelka

erich.pawelka@gesundheitsverbund.at

Theresa Mader

theresa.mader@gesundheitsverbund.at

Sara Omid

sara.omid@gesundheitsverbund.at

Hasan Kelani

n1542239@students.meduniwien.ac.at

Sebastian Baumgartner

sebastian.baumgartner@gesundheitsverbund.at

Sarah Ely

sarah.ely@meduniwien.ac.at

Wolfgang Hoepler

wolfgang.hoepler@gesundheitsverbund.at

Bernd Jilma

bernd.jilma@meduniwien.ac.at

Franz Koenig

franz.koenig@meduniwien.ac.at

Hermann Laferl

hermann.laferl@gesundheitsverbund.at

\author{
Marianna Traugott \\ marianna.traugott@gesundheitsverbund.at \\ Michael Turner \\ michael.turner@gesundheitsverbund.at \\ Tamara Seitz \\ tamara.seitz@gesundheitsverbund.at \\ Christoph Wenisch \\ christoph.wenisch@gesundheitsverbund.at \\ Alexander Zoufaly \\ alexander.zoufaly@gesundheitsverbund.at \\ 1 Department for Infectious Diseases and Tropical Medicine, \\ Kaiser-Franz-Josef Hospital, Kundratstraße 3, 1100 Vienna, \\ Austria \\ 2 Department of Clinical Pharmacology, Medical University \\ of Vienna, Vienna, Austria \\ 3 Section for Medical Statistics, Medical University of Vienna, \\ Vienna, Austria
}

